\section{Hacia relacionismos integrales, la extensión entre la investigación}

\author{
Paula Contino
}

continopaula@yahoo.com

\section{Mariela Daneri}

mares@arnet.com.ar

Universidad Nacional de Rosario, Argentina.
Investigación y extensión universitaria / Perspectivas
RECEPCIÓN: 29/07/18

ACEPTACIÓN FINAL: 26/09/18

\section{Resumen}

Para deliberar en términos relacionales, la palabra "entre" nos resulta cómoda para salir de ciertos trayectos señalizados. Esta preposición se ubica en medio de dos o más cosas o en relación con ellas. El "entre" es un concepto poroso, permeable, que nos aleja de las acciones disociadas de nuestra arquitectura institucional para imaginar ámbitos articulados o enlazados que hasta el momento han aparecido escindidos. En consecuencia, el texto alude a una reflexión teórica que permita la convergencia sinérgica entre dos funciones sustantivas de la universidad, la extensión y la investigación.

Palabras clave: integralidad, rizoma, aula social, cuerpo, conocimiento.
Towards integral relations, the extension between research

\section{Abstract}

In order to deliberate in relational terms the word "between" is comfortable for us to leave certain marked routes. This preposition is made through two or more things or in relation to them. The "between" is a porous, permeable concept that moves us away from the dissociated actions of our institutional architecture to imagine articulated or linked areas that until now have appeared split. Consequently, the text alludes to a theoretical reflection that allows the synergic convergence between two substantive functions of the university, extension and research.

Keywords: integrality, rhizome, social classroom, body, knowledge.
Para as relações integrais, a extensão entre pesquisa

\section{Resumo}

A fim de deliberar em termos relacionais, a palavra "entre" resulta confortável para deixarmos certos trajetos marcados. Esta preposição se posiciona no meio de duas ou mais coisas ou em relação a elas. O "entre" é um conceito poroso e permeável que nos afasta das ações dissociadas de nossa arquitetura institucional para imaginar áreas articuladas ou interligadas que até agora pareciam divididas. Consequentemente, o texto alude a uma reflexão teórica que permita a convergência sinérgica entre duas funções substantivas da universidade, extensão e pesquisa.

Palavras-chave: integralidade, rizoma, sala de aula social, corpo, conhecimento.

Para citación de este artículo: Contino, P. y Daneri, M. (2018). Hacia relacionismos integrales, la extensión entre la investigación. +E: Revista de Extensión Universitaria, 8(9), julio-diciembre, 3-13. doi: 10.14409/extension.v8i9.Jul-Dic.7836. 


\section{Introducción}

En este artículo el lector podrá encontrar un breve desarrollo de la extensión universitaria en tres temporalidades, que van desde los procesos de modernización del siglo XIX hasta la mirada contemporánea de la integralidad, con el objeto de poner en nueva relación las funciones universitarias. Esta perspectiva de carácter integral lleva inscriptas dos premisas: la generación de conocimientos socialmente relevantes y la ecología de saberes. Esta última refiere a una contraepistemología, que desarma jerarquías y entiende al conocimiento como intervención en la realidad. Apelando a una pedagogía rizomática, el texto profundiza acerca de la irrupción de lo diverso y lo heterogéneo, una articulación transfronteriza que desborda límites y funciones y recupera, también, la dimensión de la corporalidad. Hecho que implica la con-moción y el compromiso con la vida cotidiana a través de nuevas políticas académicas que relacionen de modo perceptivo la investigación con los procesos de extensión.

\section{La extensión y sus configuraciones temporales}

Para contextualizar esta narrativa y aproximarnos al análisis que nos convoca, tal como se adelantó, apelamos de modo sintético a tres grandes temporalidades que marcaron el recorrido de la extensión universitaria en tanto se construyó como una dimensión de la educación superior.

En primer lugar, en términos históricos, nos ubicamos en el año 1871, en Inglaterra, en las Universidades de Oxford y Cambridge, cuando en forma sucedánea a la práctica fuera de los muros se acuñaba el término extensión, definido como toda labor expansiva fuera del ámbito educativo.

Esto sucedía al calor de los procesos de modernización que venían de la mano de un nuevo modo de producción, que consolidaba al capitalismo industrial en forma progresiva y permanente, junto a nuevos hábitos culturales, técnicas y tecnologías que demandan conocimientos relevantes para hacer frente al cambio epocal, fundamentalmente la clase trabajadora que se constituía en manera creciente como sujeto colectivo.

La segunda temporalidad la encontramos en la Reforma de Córdoba de 1918, que hoy alcanza su primer centenario. Aquella gesta estudiantil que superó largamente el movimiento áulico para convertirse en un hito político y social marcó para siempre la historia de la educación superior en nuestro país y en América Latina. Desde allí se sentaron las bases del modelo democrático, laico y autonómico que, a pesar de los constantes vaivenes políticos, mantiene vivo estos valores hasta nuestros días.

El tercer eje se sitúa en la contemporaneidad del siglo XXI, donde parte de nuestra comunidad académica viene trabajando en forma sistemática y orgánica por alcanzar la jerarquización y consolidación de la extensión. Sabemos que por diversos motivos (luego volveremos sobre este aspecto) a lo largo del tiempo se convirtió en una función perimetral, en especial al lado de la investigación. Pero en los últimos quince años aproximadamente se viene dando un fuerte trabajo por agendar, por materializar su tarea y avanzar hacia mecanismos de institucionalización. Durante este proceso, muy necesario y, por cierto, diverso en el concierto de universidades públicas argentinas, se debatieron en congresos, jornadas y otros ámbitos de la vida académica distintos tópicos e interrogantes, como por ejemplo:

¿Cómo se define su conceptualización y desarrollo? 
¿Qué implica su dimensión significante mientras ponemos en tensión su sentido inherente? ¿Cómo cambiamos roles históricos que se le habían otorgado para ir hacia una relación dinámica y dialogante con el medio que la contiene?

¿Cómo otorgarle densidad y protagonismo a su misión?

Estas cuestiones, entre otras, marcaron la agenda de discusión a medida que fueron transcurriendo los primeros años del siglo actual.

\section{La integralidad como praxis rizomática}

La extensión, desde su mirada contemporánea, trajo como novedad y desafío a nuestra universidad la "perspectiva integral". Este abordaje nos coloca en otro escenario; ya no estamos pensando solo en su proceso de jerarquización, creciendo pero aislada de las otras funciones, porque de ese modo estaríamos cayendo en lo mismo que cuestionamos. La noción de integralidad implica un nuevo posicionamiento político, epistémico y metodológico en la producción del conocimiento. Este cambio de perspectiva necesita enlazar las funciones de investigación-extensión de manera sensible e inteligible, para intervenir en la complejidad social en el marco de la multiplicidad de voces.

El gran reto consiste en convertir a la extensión en principio organizador de nuestra tarea para posibilitar un espacio de convergencia del tríptico misional: docencia, extensión e investigación. Se trata de avanzar de manera efectiva hacia conocimientos socialmente relevantes, basados, según Boaventura de Sousa Santos, en la "ecología de saberes"1 que permitan el descentramiento del conocimiento científico como fuente de verdad excluyente. Así podríamos entender la producción de conocimiento como un territorio en disputa que tensione las formas universalizantes y homegeneizantes de hacer y conocer, intentar la búsqueda en sus propios bordes o desbordes donde otras formas de pensar emergen, donde otra vida es soñada. En este sentido, podríamos sugerir una "pedagogía rizomática" como aquella que se desarrolla y se dispersa transversalmente, es decir, en forma colectiva aparece un proceder intersticial donde se diluyen tanto el adentro como el afuera de la institución educativa y podemos imaginar un aula social en movimiento que transcienda los límites físicos y simbólicos de nuestra universidad. En palabras de Carlos Molina Gómez:

"El adentro rígido, inflexible, normalizado, controlador y afuera lo inesperado, la pesadez del camino, la incertidumbre de una búsqueda que no tiene certezas, ni seguridades, no hay programaciones previas. El ponerse en marcha enseña que salir es maravillarse con el mundo, es disfrutar el viaje, es dejar las normas y las comodidades del adentro para gozar la vida. El viajar es la gran salida, es el camino al afuera, es avanzar a los bordes. Pero... ¿Lo que mueve el pensamiento es un adentro desestructurado o la inquietud de un afuera incalculado?". (2012:223)

\footnotetext{
1) Como propone de Sousa Santos, el pensamiento ecológico, entendido como una contraepistemología, reconoce la pluralidad de pensamientos heterogéneos y enfatiza las interconexiones dinámicas que existen entre ellos. Frente a una arraigada concepción monocultural del conocimiento, la "ecología de los saberes" concibe el conocimiento como "intervención en la realidad" más que una jerarquización de los conocimientos occidentales sobre otras formas de conocer. Ver: https://modernidadesdescentralizadas.com/actividad/retos-del-arte-global-hacia-una-ecologia-delos-saberes/
} 
La fuerza de este modo de concebir el conocimiento reside en la posibilidad de ser territorio disponible para el encuentro de otros mundos. Retomando las palabras del autor convocado y:

"entendiendo al mundo no como totalización, sino como elaboraciones vitales del existir, batallas contra el anonimato, la homogenización y la estandarización a la que someten estados y regímenes que significan representación y abstracción". (Molina Gómez:223)

Entonces, ¿qué queremos decir cuando proponemos la metáfora del rizoma como forma de concebir las ciencias y los conocimientos que estas producen cuando son capaces de articular la investigación y la extensión?

Deleuze y Guattari desarrollaron este concepto basándose en el rizoma botánico, por ejemplo, el jengibre, un tallo subterráneo que crece en forma horizontal, que emite brotes desde sus nudos. Aquí se recupera como modelo descriptivo la organización de los elementos que no sigue líneas de subordinación jerárquica sino que cualquier elemento puede incidir en otro. El rizoma crece de un centro, por eso el concepto es tomado de la estructura de algunas plantas cuyos brotes pueden ramificarse y funcionan como raíz, tallo o rama. Un rizoma está hecho de mesetas que no están en el principio ni en el final, sino siempre en el medio, en el entre. Es una región continua de intensidades, que vibra sobre sí misma y que se desarrolla evitando cualquier orientación hacia un punto culminante o hacia un fin exterior.

Por otro lado, y en contraposición con esta metáfora, otros autores utilizan la figura del árbol, el cual presenta una estructura jerárquica, su tronco es el núcleo, el centro de poder que determina el resto de las ramificaciones. Este modelo de pensamiento arborescente se basa en estructuras con lógicas binarias y dicotomías (lo bueno-lo malo; lo blanco-lo negro; lo de arriba-lo de abajo; el que sabe-el que ignora; el que siente-el que razona; el que hace-el que piensa). Aquí las raíces estructuran y dan conocimiento a saberes compartimentados, controlados y dirigidos monolíticamente. El calco y la reproducción se establecen como lógicas del conocimiento que ofrecen sus frutos en lo más alto de la cúspide. Contrariamente, en el jengibre no hay jerarquía en sus ramificaciones, no tiene un tronco principal desde donde salen las bifurcaciones sino que cada una de sus partes es diversa, heterogéna y fundamental, porque cualquiera de ellas puede generar una nueva planta.

La metáfora del rizoma permite de este modo romper con el paradigma epistemológico clásico, dado que, por una parte, niega la construcción de saberes de manera jerárquica y arborescente, emitiendo sus múltiples desgloses pero siempre desde un tronco inicial común. En su lugar, postula una forma mucho más libre de producción y articulación de saberes diferentes pero entrelazados.

"Estos conocimientos se presentarían como subterráneos, invisibles, de carácter transversal en cuanto rompen disciplinas y desequilibran sus fronteras. Cuando un rizoma se corta, o se intenta parar, su crecimiento caótico lo hace aparecer en otro espacio, a través de otro hueco, siempre desborda. Los rizomas se basan en la cartografía y la producción diferencial, es decir en un mapa constantemente construido, desplegado y reapropiado por otros, siempre desmontable, apropiable, transferible, alterable o modificable, con múltiples salidas y líneas de entrada." (Montes, 2015:1) 
Por otra parte, y de manera principal, la metáfora aludida no permite diferenciar "individualidades" en esa trama. Esto es, no presupone un sujeto observador separado, individualizado y distinto de la realidad que observa, sino que insta a concebir al "observador" como zambullido en una red, en constante flujo y cambio, relaciones de interdependencia en las que las operaciones de observación (operaciones realizables en el campo de la investigación) no son algo separado o escindido del resto de las acciones en que se mantiene y desarrolla vivo el entramado rizomático. Entonces, el rizoma siempre es colectivo y múltiple. Así, Ana Paula Montes continúa su planteo y agrega:

"podemos comprobar que los rizomas no son sólo una imagen literaria, sino una forma comportamental de situar y comprender el conocimiento de forma alternativa, y con ello situarlo en lo político. Son elementos de un múltiple fluir intermedio: entre sistemas, individuos, reglas, territorios, su velocidad y fluidez se basa en estar en el medio, en ser medio". (Montes:2)

Tal como pensamos la integralidad, los rizomas conectan, potencian y multiplican dando espacio a la emergencia, el desborde, lo inesperado, lo diverso pero también lo colectivo. Por ello, una educación de este estilo se demarca como performativa, creativa y transfronteriza, entrama discursos, posiciones y visiones, subvierte normas que permitan desbordar instituciones.

En este sentido, la interrelación disciplinar es vital para transcender los temas y pasar a los problemas en todo su cuerpo y densidad. Como hemos planteado, la realidad emerge compleja, acontece; pero paradójicamente, en la práctica cotidiana consolidamos la hiperespecialización no solo del campo disciplinar sino también de la incumbencia temática que guía nuestro trabajo académico. Únicamente en el ámbito del posgrado nos encontramos con profesionales de otras procedencias y podemos mirar y, quizás, comprender "la realidad" con otra profundidad, con otra trama que dé cuenta de lo vivo. Sin embargo, en la instancia del grado esto no sucede en forma sistemática, salvo en algunos trayectos académicos, de naturaleza electiva o no, que se convierten en espacios transversales de análisis y en ocasiones de intervención.

Mientras la sociedad se complejiza de manera creciente, las asimetrías recrudecen y se cristalizan en desigualdades materiales y simbólicas; las respuestas por paradigmas o por disciplinas no son suficientes para intervenir. Del mismo modo, la disociación entre investigación y la extensión se convierte en un área estéril para el conocimiento vital y fecundo que nuestra sociedad requiere.

La retórica sobre esta problemática encuentra enunciación fácil y recurrente, hay sintagmas que se repiten hasta el cansancio sobre el deber ser de nuestra universidad que evidentemente son inconducentes; necesitamos reflexionar seriamente sobre nuestras políticas académicas y su formas de implementación a través de procesos relacionantes de gestión (luego retomaremos esta idea).

\section{Entre los cuerpos}

Otro aspecto clave que es necesario incorporar al análisis es la cuestión de la corporalidad en toda su dimensión. En nuestra cultura occidental, que por supuesto incluye a nuestra cultura académica, el cuerpo también ha sido tabicado, ubicado prácticamente como instru- 
mento de la razón. Sin lugar a dudas, la perspectiva integral necesita recuperar el cuerpo en toda su complejidad para poner en diálogo legítimo la investigación y la extensión si pretendemos desarrollar profesionales con conciencia crítica y ciudadana en el marco de sus intervenciones territoriales.

La construcción de binomios o pares dicotómicos muchas veces se ha convertido en una constante a lo largo de nuestra producción de conocimientos donde los sentidos en lugar de articularse se fragmentan al igual que las funciones descriptas. Seguramente el lector podrá elegir o pensar en varios de ellos. Aquí traemos algunos que se convierten en ilustrativos ejemplos de aquello que queremos expresar:

- Universidad y sociedad, (como si nuestra institución estuviera desgajada de la sociedad que la contiene y la produce).

- Individuo y totalidad.

- Cuerpo y alma.

- Hombre y mujer.

- Mito y logo (pudiendo apelar a las figuras de Apolo y Dioniso, según la mitología griega).

- Racionalidad y emocionalidad.

En este último par, la emoción ha quedado suspendida por la fuerza del logo, hecho que ha segmentado de manera artificial nuestro cuerpo entre psiquis y corporalidad, como si fuesen ámbitos diferenciados de la condición humana.

Creemos que, tanto en los procesos de investigación como en los de extensión, si no hay un cuerpo puesto en movimiento, en comunicación con otros, es muy difícil generar con-moción que pueda construir otra materialidad, otra conciencia social. Es decir, aquello que llamamos experiencia, en tanto sedimentación de nuevas formas de comprensión y compromiso con nuestro devenir. Hay que reponer el cuerpo en su entramado complejo. La integralidad como movimiento instituyente puede colocar al cuerpo como lo que efectivamente es, el primer territorio vital que habitamos. Por eso es necesario explorarlo, develarlo, desnaturalizarlo. Pensarlo en clave de multiplicidades, de modo tal que podamos trascender y superar la dicotomía cuerpo-psiquis. El cuerpo entendido como composición se define por los afectos, su capacidad inteligible, potencia y vitalidad. Ese cuerpo - singular y colectivo- es el que puede sumergirse en la intervención social, en la pedagogía rizomática que, en definitiva, se convierte en visión integral.

No hay forma de establecer verdaderos lazos por fuera de la corporalidad que nos define. "Inscribirnos en la lógica del cuerpo y de sus fuerzas implica pensar en los desplazamientos, en las reconfiguraciones de nuevas formas sensibles e inteligentes que abren paso a la diversidad de la vida". (Cabra, 2013:9)

En palabras de Kaplún:

"es necesario recuperar para la educación dimensiones perdidas o devalorizadas, además de lo intelectual y cognitivo (necesitamos) incluir también lo afectivo, lo artístico, lo ético, lo físico y lo corporal". (2012:s/p)

Desde esta mirada, la experiencia se inscribe en el cuerpo dejando huellas particulares hacia nuevas configuraciones, hacia nuevas capacidades creativas polifónicas que nos 
permitan salir de los esencialismos dicotómicos para habitar estos procesos en toda su multiplicidad. Desafiándonos a salir de la cómoda ortodoxia que plasmó la superioridad del conocimiento científico respecto de otros saberes, bajo la pretendida neutralidad, apelando a métodos de comprobación universal y criterios excluyentes de verdad.

Esta fuerza instituyente persigue una resignificación dialogante de nuestros propios modelos de comprensión y acción.

Graciela Frigerio, una educadora extraordinaria de nuestro país, formada intelectualmente en diversos campos de las ciencias sociales, se refiere a los "oficios del lazo" para quienes intervienen y transitan los acontecimientos y vicisitudes de la vida cotidiana a través de las instituciones, incluso para aquellas que trabajan en el límite o en los bordes de la desafiliación. Es justamente la idea del lazo un clivaje necesario para hablar de integralidad, porque el lazo es lo que efectivamente acontece entre las partes, lo que liga, lo que acerca; porque no hay hecho social si no es en relación. El lazo puede, de algún manera, asegurar presencias significativas que resistan la orfandad, en especial en tiempos de intemperie, esquivos a la igualdad que necesita la vida en común.

\section{Venir entre}

Llegado este momento, aparece específicamente aquí la categoría intervención, concepto polisémico y, en ocasiones, polémico. No obstante, pretendemos recuperar el sentido etimológico de raíz latina del vocabo interventio, que quiere decir venir-entre. Otra vez el "entre" que nos permite articular, como grafía distante de las dicotomías que habitan cierta parte de nuestra arquitectura institucional.

Entendemos que si solo se piensa la intervención desde sus más tradicionales o literales interpretaciones (interposición, intercesión, intromisión, injerencia, intrusión) la polémica es clara, dado que en estos sentidos la intervención aparece como el acto de un tercero que sobreviene en relación con un estado preexistente con cierta connotación negativa, al menos intrusiva. Ahora, bien diferente es pensarlo de la mano de la tradición de la psicología social, donde la intervención se convierte en un procedimiento (pedagógico, metodológico, analítico, político y ético) aplicado a las comunidades, en una práctica cuyo objetivo es el conocimiento de las situaciones estudiadas y el posterior análisis de la dinámica del cambio que pueda derivarse de ella.

Entendida así, para los objetivos de la intervención es mucho menos interesante la rehabilitación de los organismos sociales o el tratamiento de las disfunciones que la interrogación acerca del sentido, la puesta en evidencia y la elucidación de lo que hasta ese entonces estaba oculto en los fenómenos institucionales debido al juego de los intereses y su opacidad resultante. Esta forma de comprender la intervención social, como diálogo, como instancia relacionante, se articula con estos modos de volver pensable en el acontecer social.

Entonces, desde esta perspectiva, intervenir es crear mapas, territorios, líneas de fuga, espacios de resistencia, tiempos y lugares de suspensión y desaceleración de lo instituido. Poner en suspenso el sentido para la creación de significaciones nuevas, territorios nuevos, en definitiva, otras posibilidades de imaginar lo deseable.

Se presenta así el desafío de pensar la construcción del conocimiento y sostener la intervención social desde maneras asociativas y colaborativas, que admitan y reconozcan la 
diversidad de actores, que permitan recuperar experiencias más estéticas que lógicas, y más imaginativas que doctrinarias. Asumiendo un modo de vinculación heterogéneo, donde la multiplicidad de proveniencias, tanto disciplinares, geográficas, generacionales como ideológicas, pueda generar una riqueza que potencie y nutra lo vivo.

\section{Políticas del conocimiento}

En este contexto, se vuelven relevantes el diseño y la implementación de políticas académicas o de políticas del conocimiento que sean capaces en verdad de integrar procesos, sujetos, funciones (extensión e investigación) y aconteceres, a fin de no perdernos en potencia en la separación. Es decir, arribar hacia una convergencia inteligente y sensible en los instituyentes que queremos configurar. La convergencia, concepto fundamental del lenguaje digital, nos posibilita imaginar nuestra organización universitaria de manera confluente, en red o, mejor dicho, construyendo redes que tejan lazos.

Vale aclarar que cuando nos referimos al diseño e implementación de políticas, en este caso académicas, no lo planteamos de modo sucedáneo, es decir, primero el diseño y luego la implementación como fases lineales y diferenciadas de la política estatal en cualquiera de sus organizaciones.

En líneas generales, las políticas son diseñadas desde nociones preconcebidas, que en ocasiones solo proyectan aquello que creen necesario para un beneficiario o destinatario que, tal como lo indica su nombre, se convertirá en el destino final de una política. Mientras que los implementadores serán quienes vean la distancia efectiva entre lo pensado y las emergencias de la puesta en práctica, como también la legitimidad o no del proyecto en términos de apropiación. En este sentido, destacamos que la implementación es la carnadura material y simbólica de la política, por eso es necesario el diálogo interactuante de las funciones académicas mediado por la gestión en todas sus dimensiones.

\section{Recuperar recorridos y causalidades}

El planteo que venimos realizando hasta acá no es novedoso, y obviamente no es iniciático, creerlo sería al menos un acto de ignorancia o de arrogancia. Lo que sí es novedoso es que la extensión universitaria haya traído a la escena académica la perspectiva integral para transitar hacia el principio de la praxis saberes para la acción.

Hay múltiples referencias, tanto teóricas como metodológicas, que problematizan esta cuestión desde diferentes ámbitos de estudio. Podríamos indagar una larga historia desde el "intelectual orgánico" de Antonio Gramsci, con su teoría para la acción, hasta el "intelectual público" de Atilio Borón, quien, retomando al filósofo italiano, exhorta a los intelectuales a salir de la endogamia académica y el diálogo entre pares para avanzar hacia procesos democratizadores del conocimiento.

Por su parte, las metodologías de Investigación-Acción-Participativa, denominadas IAP, basadas en la pedagogía latinoamericana freireana, a través de distintos pensadores como Fals Borda, definen a la investigación-acción como procesos colectivos y cambian la idea de sujeto-objeto para pasar a la relación sujeto-sujeto como espacio de encuentro. En esta relación, el investigador aparece como "animador" de procesos. 
La antropología, con su aporte del método etnográfico, se transformó en un camino que permitió entender el sentido que da forma y contenido al acontecer social y es, por lo tanto, un método central para la investigación de carácter social.

El campo Ciencia-Tecnología-Sociedad (CTS), que alumbró en la década del '70, construyó este triángulo y generó uniones vitales y nuevos sentidos para la producción del conocimiento socialmente relevante.

De manera contemporánea, la comunicación social de la ciencia, una nueva línea dentro del campo comunicacional, ha trabajado sobre la ampliación de los umbrales de la ciudadanía a partir de cuestionar la generación, distribución y apropiación del conocimiento. Alejándose, del mismo modo que la extensión, de los procesos difusionistas o transferenciales.

Claramente, podríamos seguir enunciando valiosos aportes para enlazar las formas de conocer o aprehender la "realidad" que debieran dar sentido a nuestro accionar. Pero es evidente que estas contribuciones no se objetivaron en la estructura organizacional universitaria.

A lo largo del tiempo no hubo polivalencia entre la extensión y la investigación porque se establecieron jerarquías y la primera quedó ubicada de manera perimetral, como una correcta, moralmente buena o voluntariosa. Solo asociada a la práctica y fuera del reconocido ejido académico. Podríamos decir que quedó minorizada frente a la investigación, que se construyó como fuente de prestigio, casi como un fin en sí misma y no como una fuente vital y relacional para los procesos de intervención con apropiación colectiva.

Este descentramiento rizomático que trae consigo la noción integral nos aleja de la tríada saber-poder-verdad que ha acompañado a la producción cientificista y al racionalismo puro desde comienzos de la modernidad.

También encontramos otras razones por las cuales la extensión ha quedado aislada de las funciones centrales de la vida universitaria. Podemos ubicar en el tiempo el avance de las oleadas conservadoras que traen consigo políticas regresivas o restrictivas que nos distancian de los procesos democratizadores, de los valores públicos, de la ampliación de derechos. Entonces, poco tiene que hacer la extensión en medio de políticas neoliberales cuando su misión se sitúa en el compromiso social universitario desde una mirada crítica, entendiendo a la educación como un derecho humano, un bien público y social, y no como mercancía o servicio.

En esta línea, las interrupciones democráticas, o mejor dicho los golpes cívico-militares que tuvieron lugar en nuestro país entre el año 1930 a 1983, cercenaron no solo la posibilidad de un régimen democrático sino también los procedimientos básicos de la vida democrática y, por supuesto, la tarea extensionista se quedó con un campo de acción marginal o prácticamente nulo.

Estos pueden ser algunos de los motivos por los cuales esta función no se consolidó frente a la investigación; pero también existen otras razones que se vinculan en forma directa (y obviamente no aislada de los contextos sociales mencionados) con aquellas decisiones que han transformado nuestro sistema institucional. En este caso, podemos referimos al programa de incentivos centrado en la investigación que —más allá del interés legítimo y necesario de dicha función para el desarrollo de conocimientos- desde la década de los '90 se convirtió, entre otros aspectos, en la posibilidad de aumentar el precarizado salario docente. Al mismo tiempo, esta exigencia encontró ejecución en los procesos de categorización que se edificaron sobre una escala jerárquica para entrar al sistema. 
En forma encadenada, esta situación impactó en la carrera docente, que hoy se expresa en el nuevo formato del $\mathrm{CVar}^{2}{ }^{2}$ donde se evalúa y pondera la tarea docente.

En este camino, queremos señalar un hito sustancial, la homologación del Convenio Colectivo de Trabajo en el año 2014, hecho que por sus características permitió el ingreso a carrera a muchos docentes que desde hacía años ejercían como tales sin acceder a la totalidad de los derechos como trabajadores de la educación. Y señalamos este parteaguas porque dicha carrera se convierte en un punto clave para consolidar no solo la extensión sino su relación con la investigación.

Aquí queremos destacar que en la Universidad Nacional de Rosario, a propósito del mencionado Convenio, el Consejo Superior aprobó el año pasado la propuesta de la Secretaría de Extensión Universitaria para crear el Reglamento de Acreditación de Programas, Proyectos y Actividades de Extensión por fuera de las convocatorias institucionalizadas. Esta normativa tiene como finalidad validar toda la tarea que se realiza en ese sentido y obtener polivalencia con los proyectos de investigación que son fundamentales en la carrera académica de todos los docentes. Esto podría crear un sistema de distribución que equilibre las funciones tal como plantean los desafíos actuales. Este hecho se vería potenciado si se lograsen modificar ciertos aspectos del CVar que, si bien contiene en su grilla a la extensión como función, necesitaría ajustes para contener de manera fehaciente la labor que lleva adelante el docente en todo este campo diverso.

Sin dudas la riqueza estaría en una evaluación que ponderara no solo la extensión e investigación en igualdad de condiciones sino también ambas misiones en diálogo efectivo para la acción territorial.

\section{Reflexiones finales}

Sin ánimo de concluir o dejar una premisa delineada sobre lo expuesto, creemos que quizás sea momento de apelar a ciertas preguntas o reformular viejos interrogantes que eviten las respuestas fáciles y ensayadas que, a su vez, simplifiquen el análisis.

Entonces, insistimos con la incomodidad de la pregunta:

¿Cómo forjamos condiciones de posibilidad para construir el lazo social en términos de producción de conocimiento?

¿De qué manera dialogamos con otros sujetos sociales que forman parte de nuestra contemporaneidad?

¿Cómo volvemos pensable lo que acontece en el territorio a través de nuevos modos de intervención e investigación o investigación-acción?

¿Cómo creamos procesos inteligibles y sensibles a la hora de conocer o producir conocimientos?

La sensibilidad propia de la afectividad es una cualidad que no ha sido incorporada de manera efectiva a la tarea académica e incluso desde ciertos imaginarios ha quedado asociada a la debilidad o, al menos, a la falta de fortaleza. Esta metodología relacionante que

2) El CVar es un Registro Unificado y Normalizado a nivel nacional de los Datos Curriculares del personal científico y tecnológico que se desempeña en las distintas instituciones argentinas. Ver: http://www.mincyt.gob.ar/accion/ cvar-6467 
proponemos como parte de la mirada integral requiere de la sensibilidad para registrar a esos "otros" si queremos una tarea integrada de nuestra labor académica. No hay posibilidad de generar compromisos o lazos de solidaridad —en tanto procesos identificatorios con las causas que nos afectan y las que no lo hacen- si no somos capaces de con-movernos. Movimiento que se transforma en acción que impulsa y necesita del proceso de investigación para volver pensable lo que aconteció o, a la inversa, investigar para luego intervenir con la legitimidad necesaria que nos da conocimiento y, por lo tanto, la apropiación posterior que necesita la acción.

De este modo, podemos cartografiar las huellas que dejamos en cada uno de los territorios que decimos atravesar y recorrer de múltiples formas. El tema es considerar qué tipo de huellas dejamos y cuáles son las deseables. Porque siempre que intervenimos, más allá de los resultados, dejamos marcas de nuestro andar que requieren una mirada reflexiva que nos aleje de dualidades y nos acerque a las ecologías que recrean interdependencias más vitales.

\section{Referencias bibliográficas}

Cabra, N. (2013). Comunicación como trasmutación de cuerpos. Recuperado de

https://es.scribd.com/document/39632062/Comunicacion-como-transmutacion-de-cuerpos - 2013

Frigerio, G. (2017). Trabajar en Instituciones. Los oficios del lazo. Buenos Aires: Noveduc.

Gramsci, A. (1967). La formación de los intelectuales. México: Grijalbo.

Guattari, F. y Deleuze G. (1984). Mil mesetas. Capitalismo y esquizofrenia. Valencia: Pre-textos.

Kaplún, G. (2012). La integralidad como movimiento instituyente en la universidad. InterCambios, (1). Disponible en: http://intercambios.cse.edu.uy

Molina Gómez, C.A. (2012). Otra pedagogía rizomática como desplazamiento de pensamiento. Revista Colombiana de Educación, (63). Bogotá.

Montes, A.P. (2015). AULABIERTA: Un modelo colectivo de pedagogía rizomática. Recuperado de https:// eacvvcae.wordpress.com/p-t/rodrigo-javier/aulabierta-un-modelo-colectivo-de-pedagogia-rizomatica/ 